ISSN: 2277-3754

ISO 9001:2008 Certified

International Journal of Engineering and Innovative Technology (IJEIT)

Volume 10, Issue 2, August 2020

\title{
A Modified Normalized Channel Estimation Method for Efficient Compressive Sensing Based SWA by 2D Frequency Characterization
}

\author{
Amrita Kudesia $^{1}$, Meha Shrivastava ${ }^{2}$
}

Truba Institute of Engineering \& Information Technology, Bhopal, India

\begin{abstract}
Shallow or Deep-water acoustic (SWA) is widely being used for under water marine communications. The most essential part of the SWA is multipath channel modeling. Due to the huge data and limited bandwidth under water environment the compressive sassing based channel estimation is widely adopted for communication. In this paper a SWA channel estimation method is presented using modified compressive sensing (CS). The new method assumes the higher SNR values and respective higher window sizes maintaining the compressive time and frequency sparsity.Paper in first pass validates the existing SWA channel estimation methods using CS methods. Then during second pass performance is evaluated under the modified channel normalization based non uniform multipath fading based channel estimation and space recovery. The numbers of iteration counts are also compared for performance evaluation. It is found that modifying the channel realization can improve the efficiency of CS based channel estimation for SWA environment.
\end{abstract}

Key words: Shallow Water Acoustic, Channel Estimation, Compressive Sensing, Sparsity Frequency characterization.. Multipath fading

\section{INTRODUCTION}

Shallow Water Acoustic (SWA) is also known as Under Water Acoustics (UWA). Acoustics based communication systems are common in under water environments [1]. As the underwater environment is not uniform with depth thus there are various multipath components between the transceivers. Due to the presence of the energy transients the channel suffers from the huge frequency sparsity in the underwater conditions. This frequency diversity in this paper are modeled at higher Doppler frequencies using compressive sensing techniques.

The SWA channel can be modeled using the sparsity ass it contains few non-zero samples over larger sample space. Due to the sparse nature the underwater channel can be easily modeled using compressive sensing [2], [3] Due to the increasing data transmission demand the sparse solutions became popular over last two decades. The main advantages of sparse CS are that it is highly scalable over huge data dimensions and therefore less susceptible to noise. The challenges under the multipath fading water environments are given in the Figure 1.

Manuscript received: 25 July 2020

Manuscript received in revised form: 18 August 2020

Manuscript accepted: 05 September 2020

Manuscript Available online: 15 September 2020
Multipath components in underwater are present due to the reflection scattering or refraction of acoustic signals as shown in Figure 1. This is significant to reduce signal strength.

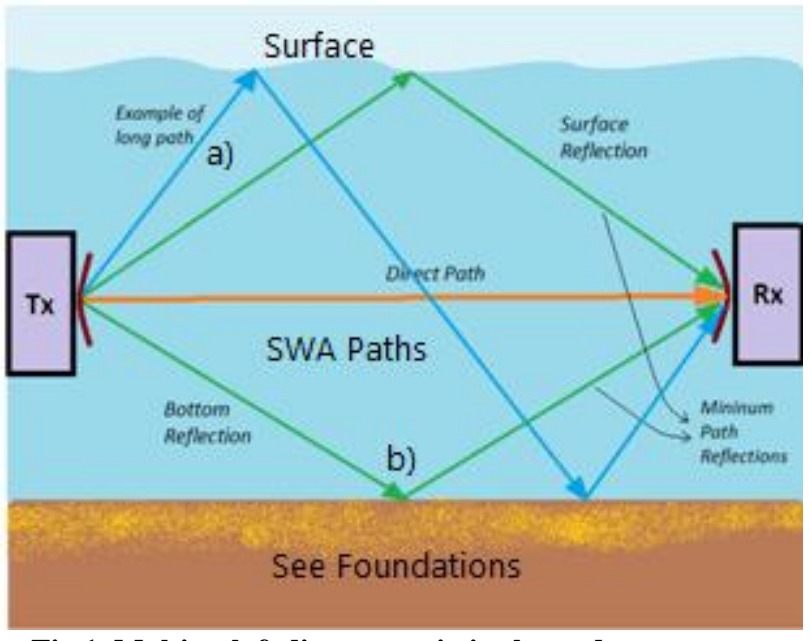

Fig.1. Multipath fading scenario in the underwater environment,

The acoustic waves are reflected back due to the bouncing of waveform the surface or foundation of the ocean. The surface of the ocean behaves like a diffuse reflector. These reflections may reduce the acoustics strengths due to dynamic multipath channel response at the receivers. These reflections are common and certain under the shallow or deepwater environments. This phenomenon is more complex in shallow water as frequency of light follows greater diversity in this environment with depth. The multipath delay spread is exceeded to wide range of around 200 delay taps. To understand the SWA scenario the Figure 2 explains phenomenon of delay spread in underwater environments.

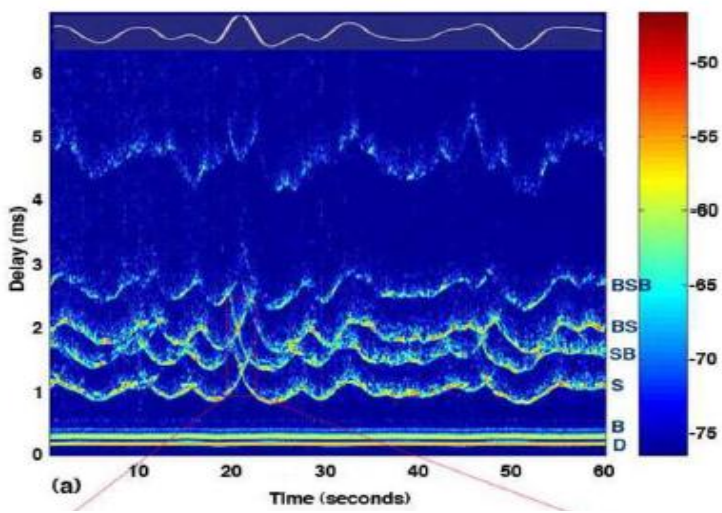

Fig.2.Delay spread in Shallow water enginemen 


\section{ISSN: 2277-3754 \\ ISO 9001:2008 Certified \\ International Journal of Engineering and Innovative Technology (IJEIT) \\ Volume 10, Issue 2, August 2020}

Due to these phenomenon's the SWA study is still a challenging problem in hand. There were numerous modeling methods available in the literature for shallow or under water acoustic channel estimation [1, 6]. Enhancing the accuracy of the SWA for CS based channel estimation is still a challenging problem in hand. Therefore this paper addressed problem of SWA under the higher expected SNR performance of existing methods over wide window dimensions without changing the CS rates.

In this paper the experimental modeling and study is presented for evaluating the effect of higher noise level and the varying the window size of the sample space over the compressive sensing based channel estimation based methods.

Rest of the paper is organized as follows: In Section II reviews of existing SWA methods of Channel estimation are discussed. Section III mathematical model for frequency selective space channel estimation method is presented. In section IV the validation of the MSE for the Existing channel estimation in CS sparse channel is presented. Section V the proposed channel modeling is presented using the normalization. Then VI section results of proposed modified CS based channel estimation method for SWA communication are presented. Section VII concludes the work and future prospects are discussed.

\section{RELATED WORK}

There were many related research found for the estimation of the SWA channel efficiently. Numerous methods are available in the literature for modeling the shallow or under water acoustic channel estimation [1, 2 and 6]. The classifications of the SWA channel modeling methods are given in the Figure 3.

The history of the SWA started long back during World War 2, when army engineer's noted that shallow water sonar broadband waves had frequency dispersion over the strange curve. This was noted that there is delay based on low medium and high frequency at the receiver. Low frequencies arrives first them followed by other two, thus making the scenario difficult to model, There are three ways of channel modeling based on time frequency and Doppler spreads as shown in the Figure
3.Naushad Ansari et al [1] have defined the three different concept of the Compressive Sampling (CS) based 2D frequency characterization for SWA channel estimation. They have presented the mathematical modeling of SWA channel sung the frequency characterization model. They stated that using 2D frequency domain is advantageous to distinguish between slow varying and highly transients SWA channel at higher frequencies. They have demonstrated non uniform compressive sensing in 2D domain.

Naushad Ansari et al [3] have recently presented the energy efficient method for the SWA channel estimation using the 2D Frequency modeling. They have explained the modeling of CS based $2 \mathrm{~d}$ frequency modeling then demonstrated the energy efficient approach of estimation. Samar Kaddouri et al [3] have presented an estimation method for high frequency sparse and dynamic underwater acoustic channel (UWAC) considering MIMO transceiver. They stated that due to time varying nature of UWAC the data rates are significantly limited. José S. et al [8] have presented the underwater noise model as case of the SWAC system. They have presented the SER estimation for noise models.

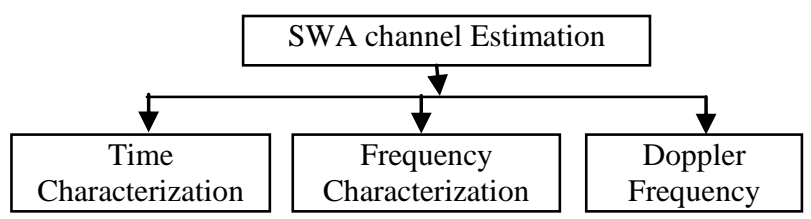

Fig.3. Classification of the SWA channel estimation methods

The methods based on the sparse recovery are frequently tested. The Compressive sensing (CS) based methods are now popular for the SWA as in the Ref. [9 and10]..CS based estimation methods gives the solution to problem of signal recovery under the underdetermined SWA system with linearity over the sparsity constraint. With the development of the adaptive CAS based signal processing and sparse recovery methods for the UWAC as in [6, and 14] encourages and became pooler to be used for UWCA. The summary of the related works and comparison SWA methods for channel modeling and sparse recovery sung CS are presented in the Table 1.

Table 1. Comparison of existing methods

\begin{tabular}{|c|c|c|c|}
\hline Author/ reference & $\begin{array}{c}\text { Method } \\
\text { Used }\end{array}$ & Area of Work & Descriptions \\
\hline Naushad [1] & $\begin{array}{c}\text { 2D frequency characterization } \\
\text { using CS }\end{array}$ & $\begin{array}{c}\text { SWA channel } \\
\text { Estimation }\end{array}$ & $\begin{array}{c}\text { Present modified CS sparse recovery using } \\
\text { 2D frequency characterization based } \\
\text { channel estimation. }\end{array}$ \\
\hline $\begin{array}{c}\text { Naushad } \\
\text { Ansari [3] }\end{array}$ & $\begin{array}{c}\text { Energy efficient SWA by 2D } \\
\text { frequency characterization }\end{array}$ & $\begin{array}{c}\text { SWA channel } \\
\text { estimation }\end{array}$ & $\begin{array}{c}\text { Energy efficient channel estimation for } \\
\text { sparse recovery for SWA }\end{array}$ \\
\hline $\begin{array}{c}\text { A. S. Gupta et al. } \\
{[14]}\end{array}$ & $\begin{array}{c}\text { Sparse channel coefficient for } \\
\text { SWA }\end{array}$ & Time varying SWA & $\begin{array}{c}\text { CS for sparse recovery under time varying } \\
\text { SWA channel. }\end{array}$ \\
\hline Nisha et al [15] & OFDM channel estimation & $\begin{array}{c}\text { OFDM transceiver } \\
\text { channel estimation }\end{array}$ & $\begin{array}{c}\text { Sparse channel estimation concept designed } \\
\text { for OFDM transceiver }\end{array}$ \\
\hline Our proposed work & $\begin{array}{c}\text { SWA 2D frequency } \\
\text { characterization using CS }\end{array}$ & $\begin{array}{c}\text { Modified CS channel } \\
\text { estimation }\end{array}$ & $\begin{array}{c}\text { 2D frequency characterization based } \\
\text { modified CS for SWA channel estimation. }\end{array}$ \\
\hline
\end{tabular}


ISSN: 2277-3754

ISO 9001:2008 Certified

International Journal of Engineering and Innovative Technology (IJEIT)

Volume 10, Issue 2, August 2020

\section{MODELING OF 2D FREQUENCY CHARACTERIZATION BASED CS CHANNEL ESTIMATION}

In paper the method of 2-D frequency characterization $[1,3]$ is used for the modeling the SWA channel SPACE08 [1].Letter paper uses this model for evaluating the modified window length effect at higher SNR level. The acoustic signal transmission is considered for underwater communication with multipath delays. The non-uniform SWA channel is consider for the representation for sparse sampling and measurements over the transient channel. Paper addressed two types of SWA channels over multipath environment as transients with high energy and slow varying channels.

The high frequency transient introduced due to interference between multipath components or dynamic scattering events. Due to their energy distribution these high frequency transient offers sparse nature. while the slow transients exists during the direct path of arrival.

\section{A. Channel Estimates Experimental Setup from Field Experiments}

Numerical experiments presented in the current research work is supported channel simulations derived from two independent sources: An independent channel simulator proposed considering the same setup of the multipath model commonly encountered in shallow water acoustic communications, considering the SPACE08 data as in [2]. During this experiment setup experimental field data was collected from shallow water at the depth of around $15 \mathrm{~m}$ within the range of $200 \mathrm{~m}$ of ocean floor area considered relatively flat and considering the constant water column temperature [2].

\section{B. Channel Representation}

The frequency-selective channel is considered as nonuniform compressive sampling model, the model is designed with 2D frequency characterization. This approach converts the channel estimation problem to nonuniform sparse recovery case using .2D Fourier transform. Let us define the following initial simulation parameters as

Table 2. Simulation Parameters Initialization

\begin{tabular}{|l|l|l|}
\hline \multicolumn{1}{|c|}{ Description } & Parameter & Range \\
\hline Numbers of total delay taps; & $\mathrm{K}$ & 200 \\
\hline $\begin{array}{l}\text { Number of total Doppler } \\
\text { frequencies }\end{array}$ & $\mathrm{L}$ & \\
\hline Index of Delay tap, & $\mathrm{k}$ & $\mathrm{K}=0,1 \ldots, \mathrm{K}$ \\
\hline Doppler frequency & $\mathrm{f}$ & $0,1, \ldots \mathrm{L}-1$ \\
\hline Delay frequency & $\mathrm{x}$ & \\
\hline Omega index & $\omega$ & $0,1, \ldots, \mathrm{K}-1 ;$ \\
\hline $\begin{array}{l}\text { Cannel impulse Realization at } \\
\mathrm{i}^{\text {th }} \text { time at kth delay tap }\end{array}$ & $\mathrm{H}_{\mathrm{i}(\mathrm{I}, \mathrm{k})}$ & Length $(\mathrm{K})$ \\
\hline $\begin{array}{l}\text { Channel matrix in 2D } \\
\text { frequency }\end{array}$ & $\mathrm{U}(\mathrm{I}, \mathrm{k})$ & $\mathrm{As} \mathrm{H}$ \\
\hline
\end{tabular}

These parameters are used to model non-uniform CS based sparse recovery method exploiting non-sparse structure for the higher frequencies at 2D frequency characterization [3] Let the exponential input signal is given as

$\begin{array}{rlrr} & \left.x(i, w)=e^{j 2 \pi i w} / k\right) & (1) \\ \text { Respective delay } & \text { frequencies } & \omega & \text { renging }\end{array}$ from $\omega=0_{s} 1_{x} . K$ These sub bands are designed easily with the use of frequency selective methods. Sample the channel using Doppler frequencies with the impulse response $\mathrm{H}(\mathrm{i}, \mathrm{k})$ the output channel is modeled as

$$
\begin{aligned}
& y[i, \omega]=\sum_{k=0}^{K=1} H\left(i_{x} j\right) x\left(i-k_{x} \omega\right) \\
& =e^{j 2 \pi i w / k)} \sum_{k=0}^{K=1} H\left(i_{x} j\right) e^{-j 2 \pi i w / k)} \\
& y[i, \omega] * e^{\left.-j 2 \pi i \omega_{j} / k\right)} l=\sum_{k=0}^{K=1} H(i, j) e^{-j 2 \pi i \omega / k)}
\end{aligned}
$$

Taking 1D Fourier transform of Doppler frequencies, it gives

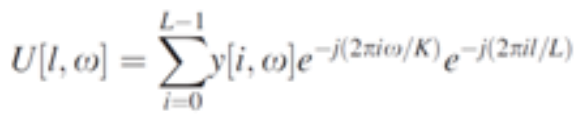

This is the 2D Fourier transform of the channel $\mathrm{H}$. in the matrix or vector form this is written as

$$
U=F_{1} H F_{2}=\varphi H(6)
$$

Where, $\mathrm{U}$ is a matrix of size $L x$, the $\mathrm{H}$ is a matrix representing channel impulse response $\mathrm{H}[\mathrm{i}, \mathrm{k}]$, the matrix $F_{1}$ and $F_{2}$ are Fourier transform matrix and $F$ is the symbolic representation to the $2 \mathrm{D}$ Fourier transform. Equation depicts channel recovery is posibel by inverse Fourier trasform for noise free envirenment. This has motivated us to explore CS based channel recovery in the proposed framework.

\section{CS BASED RECOVERY AND JUSTIFICATION}

The AWGN channel noise remains AWGN under the Fourier consideration. Thus under the presence of the noise the Eq. [6] can be re written as

$$
U_{n}=\varphi H+N
$$

Where, the $\mathrm{N}$ is the AWGN noise added to system during channel. The Figure 4 presents the example of the delay tap amplitude of the Fourier spectrum. It can be observed that channel having sparse nature of the high amplitude activity dominating over lower diffused spread of the smaller taps.

Therefore it is proposed to estimate the channel realization matrix $H$ with the $\mathrm{CS}$ based filtering of noise considering the sparse nature of $U_{n}$. The filtering problem is mathematically modeled as minimization problem as;

$$
\min _{\mathbb{U}_{n}}\left\|U-U_{n}\right\|_{2}^{2} \quad \text { Subjected to }\|U\| \leq \tau
$$

Where, trepresents the measure of sparsely of $U$ and is set to $0.5 \sqrt{L K}$. The signal to noise ratio of channel is modeled as; 
ISSN: 2277-3754

ISO 9001:2008 Certified

International Journal of Engineering and Innovative Technology (IJEIT)

$$
S N R=10 \log _{10}\left(\frac{\frac{1}{L K} \sum_{i=0}^{L-1} \sum_{j=0}^{K-1}|H(i, j)|^{2}}{\sigma_{n}^{2}}\right)
$$

The normalized mean square error NMSE of estimated channel response $H_{\theta}(i, j)$ is calculated as.

$$
\text { NMSE }=10 \log _{10}\left(\frac{\sum_{i=0}^{L-1} \sum_{j=0}^{K-1}\left|H(i, j)-H_{e}(i, j)\right|}{\sum_{i=0}^{L-1} \sum_{j=0}^{K-1}|H(i, j)|^{2}}\right)
$$

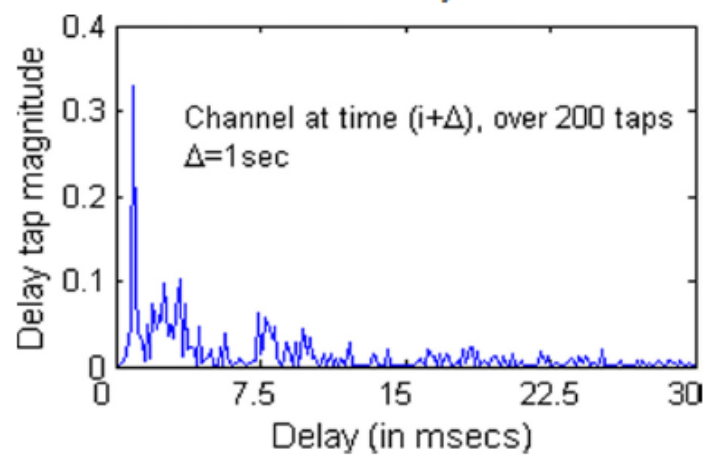

Fig.4.Example of delay tap amplitudes of the channel estimates at time $(t+\Delta)(\operatorname{Ref}[2])$

\section{A. Validation of the Existing SWA CS method}

For validation the normalized MSE is calculated using the basic CS technique [Ref. section II C of [1]] as given in Eq. 8 above. The Monte Carlo iteration is used with 100 iterations only for every noisy simple considering different lengths of Window.

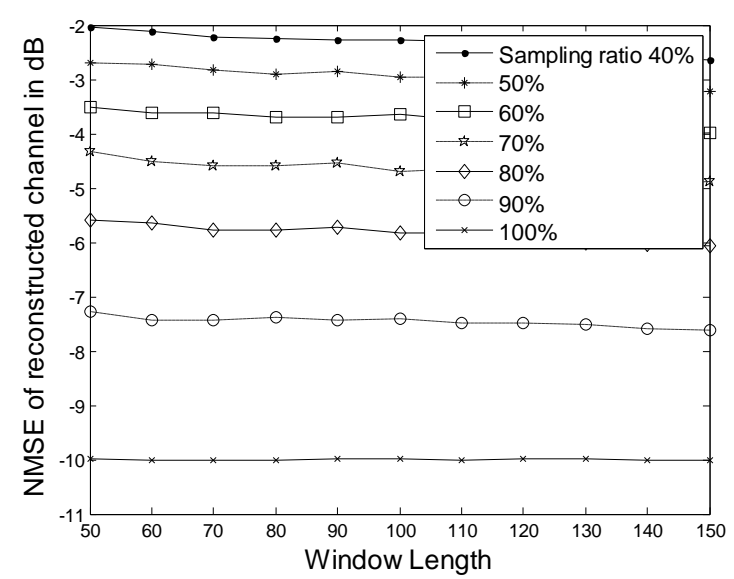

Fig.5.Validated MMSE reconstructed with CS channel evaluated estimation method of [2]

\section{PROPOSED MODIFIED CS BASED CHANNEL ESTIMATION WITH PRIOR INFORMATION.}

In this section the non uniform CS is considered with certain prior information in addition the sparsity of $U$ is applied only on specific subspace slot of Tc considering the maximum sparcity overU. This can inturn increase the performance of channel estimation. Additionally the $U$ space is sub divided to two non overlapping subspaces $S_{1}$ and $S_{2}$ :

1. Subspace Sirepresents the worst case of the $T E$ under sparsity and partial sampling. This subspace is more noisy and sparse, thus having multiple reflections from rough sea bottom. Signal contains spikes wave events, and low energy attenuated multipath arrivals.(b),

2. Subspace ${ }_{2}$, is less noisy subspace supports time frame $\mathrm{T}$, containing relatively stable and higher energy transients that occupy low Doppler regions.

The proposed CS based modified sparse channel estimation considering prior information is now mathematically formulated as,

$\min _{\mathbb{U}_{n}}\left\|U_{S u b}-R_{T^{u}} U_{n_{W}}\right\|_{2}^{2}$ Subjected to $\left\|U_{W_{T} u}\right\| \leq \tau(11)$

In this paper as a modification the channel is formulated using the normal random realization function and the window size $\mathrm{W}$ is expected to increased from 100 to 150 to 250 . Change and the sparsity range is kept the same.

The validations of the results for the modified CS method are given in the Figure 6 for the 200 Monte carol simulations. During the validation the widow length is kept the same as in [2].As the channel is randomly initialize thus the slight variations will be observed in NMSE curves every execution.

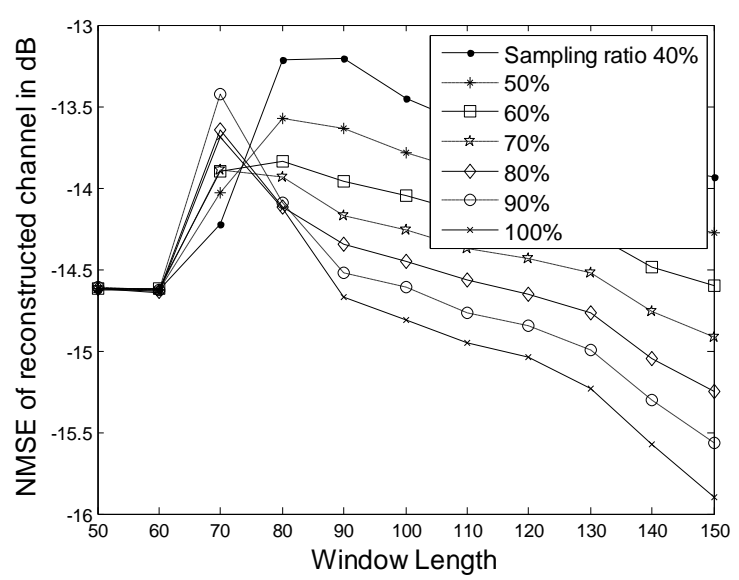

Fig.6.Validation of the MMSE achieved with the modified CS based channel estimation by [2]

\section{RESULTS AND DISCUSSION}

Section presents the comparison of experimental results for various experiment performed over the modified CS based estimation of SWA communication. During the experiment the higher values of the SNR is considered and is raised from 70 to 100 .

The results are plotted in the Figure 7 for the MMSE error recovered with modified CS based method with prior information considering the higher window length of WL 50::150 at the higher SNR level of 100. The corresponding results are plotted for window length of 20.100 in the Figure 8. For 110 SNR.

Comparing the Figure 7 with the Figure 5 . It can be observed the with the proposed modifications ad at higher SNR the NMSE is improved from -10 to -14 for both the widow sizes. Thus NMSE is converging netter with the 
ISSN: 2277-3754

ISO 9001:2008 Certified

International Journal of Engineering and Innovative Technology (IJEIT)

Volume 10, Issue 2, August 2020

proposed modified CS based parametric evaluation of the modified channel estimation.

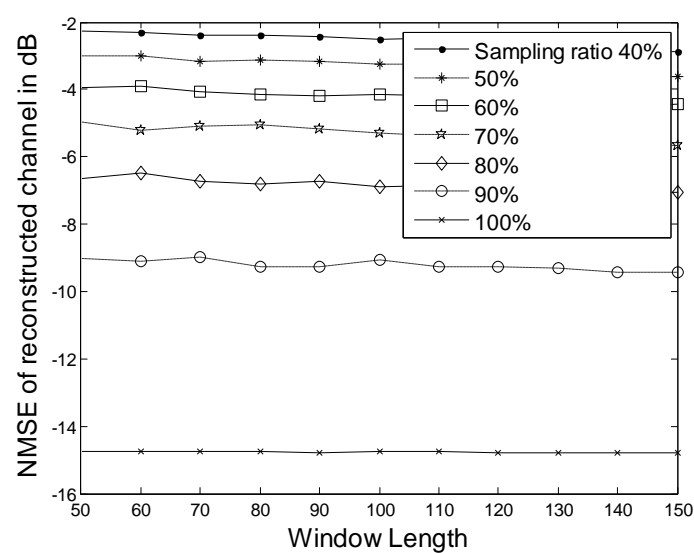

Fig.7.Results of MMSE error recovered with modified window length of WL 50::150 at the higher SNR level of 100

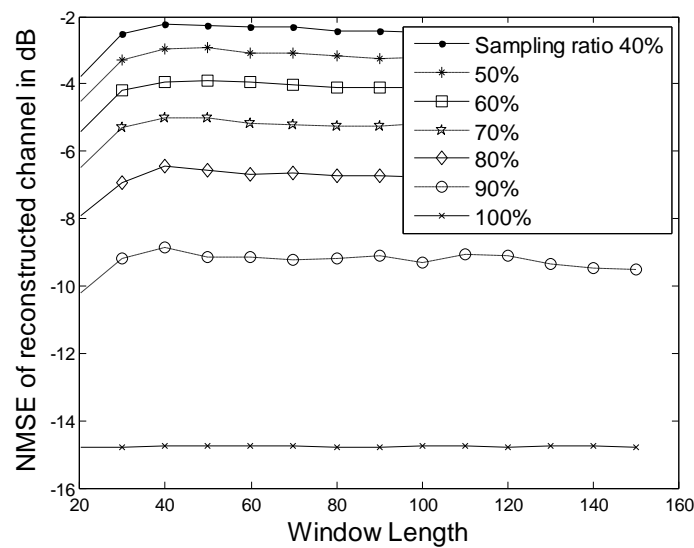

Fig.8.Results of MMSE error recovered with modified window length of $W L=20: 100$ at the higher SNR level of 100

As an another experiment NMSE results are plotted for the channel estimation at keeping the $5 \mathrm{~dB}$ SNR considering the modified sparse CS, and the support period $\mathrm{T}$ is zero and only first five non-zero Doppler frequencies considering the prior information assumption. The simulation results of the MATLAB are shown in the Figure 9a) and Figure 9 b) respectively for existing and proposed methods.

It can be observed from the Figure 9 that the result of the proposed method converges to -12.5 to -15.5 with increase in the SNR range of 100.

\section{CONCLUSION}

This Paper presents the modified CS based channel estimation method for the SWA communication considering the higher SNR range and compensating by higher widow range. The window size is increased from 100 to 150 , and the SNR is increased to 70 to 100 . The channel is realized using the modified normal random distribution. It is found that the higher SNR and widow size minimizes the NMSE performance for the same spare range of the sampling. The paper is significant contribution for evaluating the performance of the SWA channel considering the 2D frequency characterization in Fourier transform domain.

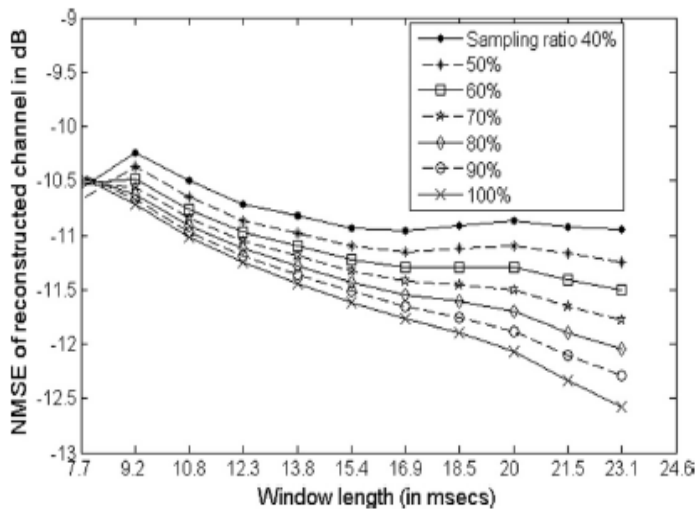

a) Result of [2]

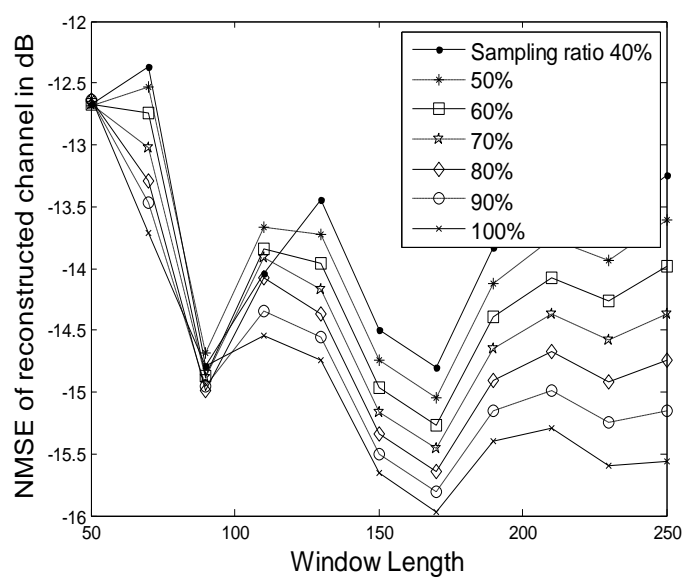

b) With proposed modified method

Fig.9.The results of the NMSE with proposed method at the Window $50: 20: 250$

\section{REFERENCES}

[1] Naushad Ansari, Anubha Gupta, Ananya Sen Gupta, "Underwater Acoustic Channel Estimation via CS with Prior Information", IEEE International conference OCEANS Aberdee UK October 2017.

[2] Naushad Ansari, Anubha Gupta, Ananya Sen Gupta "Shallow water acoustic channel estimation using twodimensional frequency characterization", international Journal .of Acoustical Society of America 140 (5), November 2016

[3] Samar Kaddouri, Pierre-Philippe J. Beaujean, Pierre-Jean Bouvet, "High-Frequency Acoustic Estimation of TimeVarying Underwater Sparse Channels Using Multiple Sources and Receivers Operated Simultaneously", Underwater Wireless Communications And Network IEEE ACCESS 2019.

[4] Jingjing Wang, Zhanjiang Yan, Wei Shi, Xinghai Yang, "Underwater Acoustic Sparse Channel Estimation Based on DW-SACoSaMP Reconstruction Algorithm”, published IEEE Communications Letters 2019.

[5] Chunguo Li, Kang Song, Luxi Yang, " Low computational complexity design over sparse channel estimator in underwater acoustic OFDM communication system", Journal of IET Communications, Vol. 11 Issue 7, pp. 1143$1151,2017$. 
ISSN: 2277-3754

ISO 9001:2008 Certified

International Journal of Engineering and Innovative Technology (IJEIT)

Volume 10, Issue 2, August 2020

[6] N. Ansari, A. Gupta, and A. S. Gupta, "Physics inspired cs based underwater acoustic channel estimation," in Signal and Information Processing (GlobalSIP), 2015 IEEE Global Conference on. IEEE, 2015, pp. 1106-1110.

[7] Qarabaqi, P., Stojanovic, M.: Statistical modeling of a shallow water acoustic communication channel. In: Proceedings of the Underwater Acoustic Measurements Conference, Nafplion, Greece, 2009.

[8] José S. G. Panaro, Fábio R. B. Lopes, Leonardo M. Barreira, Fidel E. Souza, "Underwater Acoustic Noise Model for Shallow Water Communications", IEEE SBrT'12, 13-16 DE SETEMBRO DE Brasilia 2012.

[9] D. L. Donoho, "Compressed sensing," IEEE Trans. Inf. Theory 52(4), 1289-1306 (2006.

[10] E. J. Candes and Y. Plan, "A probabilistic and RIPless theory of compressed sensing,"IEEE Trans. Inf. Theory 57(11), 7235-7254 2011.

[11] B. Li, S. Zhou, M. Stojanovic, L. Freitag, and P. Willett, "Multicarrier communication over underwater acoustic channels with nonuniform Doppler shifts," IEEE J. Ocean. Eng. 33(2), 198-209 2008.

[12] Ebrahim Baktash, Mohammad Reza Farjadi Nasab, Mahmood Karimi, Mohammad Javad Dehghani, "Shallow Water Acoustic Channel Modeling Based on Analytical Second Order Statistics for Moving Transmitter/Receiver, IEEE Transactions on Signal Processing, 2015.

[13] Jingxuan Xu, Hamada Esmaiel, Haixin Sun, Jie Qi, Mingzhang Zhou, Zeyad A. H. Qasem”, CS-Based Channel Estimation for Underwater Acoustic Time Reversal FBMC System", IEEE 5th International Conference on Computer and Communications, 2019.

[14] A. S. Gupta and J. Preisig, "Tracking the time-varying sparsity of channel coefficients in shallow water acoustic communications," in Signals, Systems and Computers (ASILOMAR), 2010 Conference Record of the Forty Fourth Asilomar Conference on. IEEE, pp. 1047- 1049, 2010.

[15] Nisha Sachan, Paresh Rawat, Nashrah Fatima , "Sparse Channel Estimation using Hybrid Approach for OFDM Transceiver", in International Journal of Computer Applications, Vol. 128 No 12015.

[16] A. S. Gupta, N. Ansari, and A. Gupta, "Tracking the underwater acoustic channel using two-dimensional frequency sampling," in Underwater Technology (UT), 2015 IEEE. IEEE, , pp. 1-5, 2015.

[17] Anagha V S, Remadevi M, Dr. Jibukumar M G, "Channel and Doppler Estimation in Underwater Communication", $8^{\text {th }}$ International IEEE symposium on Embedded Computing and system Design (ISED). 2019. 